\title{
Protective effects of oleanolic acid on oxidative stress and the expression of cytokines and collagen by the AKT/NF-kB pathway in silicotic rats
}

\author{
HAI-BING PENG ${ }^{1}$, RUI-XUN WANG ${ }^{2}$, HAI-JING DENG ${ }^{3}$, YONG-HENG WANG ${ }^{1}$, \\ JUN-DONG TANG ${ }^{4}$, FU-YUAN CAO ${ }^{5}$ and JIAN-HUI WANG ${ }^{2}$ \\ ${ }^{1}$ Medical Experimental Center of Jitang College; ${ }^{2}$ Functional Laboratory of School of Basic Medical Sciences; \\ ${ }^{3}$ Department of Pathogen and Microbiology, School of Basic Medical Sciences; ${ }^{4}$ Department of Clinical Medical, \\ Jitang College; ${ }^{5}$ Laboratory Animal Center, North China University of Science and Technology, \\ Tangshan, Hebei 063210, P.R. China
}

Received February 19, 2016; Accepted February 6, 2017

DOI: $10.3892 / \mathrm{mmr} .2017 .6402$

\begin{abstract}
Oleanolic acid (OA), a natural pentacyclic triterpenoid, has been reported to have several benefits and medicinal properties. However, its protective effects against silica-induced lung injury and fibrosis remain to be elucidated. The aim of the present study was to investigate the effects of OA on oxidative stress, and the expression of cytokines and collagen in silicotic rats. Male rats were induced by intratracheal instillation of silicosis $(250 \mathrm{mg} / \mathrm{kg})$, with the exception of the control group (NS). The rats in the OA group were intragastrically administered with OA $(60 \mathrm{mg} / \mathrm{kg} / \mathrm{d})$. The rats in the solvent control group were gavaged daily with $0.6 \%$ sodium carboxymethyl cellulose $(10 \mathrm{ml} / \mathrm{kg})$ solution for 56 consecutive days. The data showed that OA significantly attenuated the extent of silicosis fibrosis by histopathologic analysis of the lung tissues. In addition, oxidative stress activated by silica exposure, as evidenced by increasing of malondialdehyde content, and activities of superoxide dismutase and glutathione peroxidase in the lung, was regulated by treatment with OA. Furthermore, enzyme-linked immunosorbent assay analysis showed that OA significantly decreased the levels of tumor necrosis factor- $\alpha$ and transforming growth factor- $\beta 1$. Immunohistochemistry analysis showed that OA significantly decreased collagen types I and III. In investigating the mechanisms underlying the action of OA, it was found that OA decreased the level of phosphorylated AKT1, which in turn inactivated the transcriptional of nuclear factor (NF)- $\kappa \mathrm{B}$ in the development and progress of silicosis. In conclusion, these results suggested that
\end{abstract}

Correspondence to: Mrs. Hai-Bing Peng, Medical Experimental Center of Jitang College, North China University of Science and Technology, 20 Bohai Road, Tangshan, Hebei 063210, P.R. China E-mail: leizi19760728@sina.com

Key words: oleanolic acid, silicosis, oxidative stress, tumor necrosis factor- $\alpha$, tumor growth factor- $\beta 1, \mathrm{Akt} /$ nuclear factor- $\mathrm{kB}$, collagen the protective effects of OA were due, at least in part, to its anti-oxidant activity and its ability to decrease the expression of cytokines and collagen by modulating the AKT/NF- $\mathrm{AB}$ pathway.

\section{Introduction}

Silicosis, a form of occupational lung disease caused by the inhalation of crystalline silica dust, is characterized by silicotic nodule formation and pulmonary interstitial fibrosis (1). Occupational exposure to respirable crystalline silica dust particles occurs in sand blasting, drilling, pulverizing, cutting bricks and concrete blocks, grinding concrete and use of other pneumatic equipment (2) every year, therefore, silicosis is an occupational health concern in developing and developed countries $(3,4)$. In China, 20,000 cases of pneumoconiosis are diagnosed each year, and silicosis is the most common, fastest progressing and serious type (5). The pathological process of silicosis includes progressive inflammation, fibroblast proliferation and collagen deposition. In the initial inflammatory responses, alveolar macrophages, the first cells responded to stimuli of the body, are important. Following exposure to silica, a number of macrophages undergo apoptosis, resulting in the production of reactive oxygen species (ROS), which include hydroxyl radicals, superoxide anions, hydrogen peroxide, singlet oxygen $(6,7)$ and nitric oxide (NO) (8). The generation of oxidants results in cell and lung damage; increase the expression of inflammatory cytokines, including tumor necrosis factor (TNF)- $\alpha$, interleukin (IL)-1 $\beta$, and transforming growth factor (TGF)- $\beta$; activate cell signaling pathways, including the mitogen-activated protein kinase pathways; and phosphorylate and activate specific transcription factors, including nuclear factor $(\mathrm{NF})-\kappa \mathrm{B}$, which intensifies chronic inflammation and promotes pulmonary fibroblasts to proliferate and synthesize excess collagen (1).

Oleanolic acid (3 $\beta$-hydroxyolean-12-en-28-oic acid; OA) is a plant-derived pentacyclic terpenoid, which exists naturally in vegetable oil, food and certain medicinal herbs either as a free acid, or as an aglycone of triterpenoid saponins (9). It has 
been shown to exhibit numerous pharmacological properties, including hepatoprotective, anti-oxidative, anti-inflammatory and anticancer activities. Therefore, OA and its derivatives possess a wide range of applications (10). It has been reported that OA may exert beneficial effects on renal and liver fibrosis by activating nuclear factor, erythroid 2 like 2 (11) and has been used as an oral treatment for human liver dysfunction in China (10). However, the effectiveness of OA in the treatment of fibrotic lung diseases, including silicosis, remains to be elucidated. It has been reported that the phosphatidylinositol-3-kinase (PI3K)/Akt pathway is the most important pathway for the fibroblast to myofibroblast differentiation of normal and diseased primary human lung fibroblasts (12). The present study aimed to investigate the protective effects of $\mathrm{OA}$ in an experimental model of silica-induced inflammation and fibrosis by examining the oxidation/antioxidant system, TNF- $\alpha$, TGF- $\beta 1$, Akt/NF- $\kappa$ B and collagen.

\section{Materials and methods}

Materials. OA (purity $>99.9 \%$; cat. no. $110742-200513$ ) was purchased from the National Institutes for Food and Drug Control (Beijing, China) and was suspended in $0.6 \%$ sodium carboxymethyl cellulose for use. Crystalline $\mathrm{SiO}_{2}(\sim 95 \%$; 1-5 $\mu \mathrm{m}$ ), obtained from the National Institute of Occupational Health and Poison Control, Chinese Center for Disease Control and Prevention (Beijing, China), were subjected to grinding, and heating for at $180^{\circ} \mathrm{C}$ for $6 \mathrm{~h}$, followed by dilution with sterile saline to a concentration of $50 \mathrm{mg} / \mathrm{ml}$ in suspension, autoclaved and stored at $4^{\circ} \mathrm{C}$. Enzyme-linked immunosorbent assay (ELISA) kits, malondialdehyde (MDA), superoxide dismutase (SOD) and glutathione (GSH) test kits, and Masson's stain were purchased from Nanjing Jiancheng Bioengineering Institute (Nanjing, China). Phosphorylated (p-)AKT1 (phospho S473; cat. no. ab81283) and AKT (cat. no. ab28422) antibody were purchased from Abcam (Cambridge, MA, USA). NF- $\mathrm{B}$ antibody (cat. no. sc-8008) was purchased from Santa Cruz Biotechnology, Inc. (Dallas, TX, USA).

Animals and treatment. A total of 96 male adult Wistar rats (aged 6-8 weeks and weighing 180-200 g) were purchased from Vital River Laboratory Animal Technology Co., Ltd. (Beijing, China). The animal experiments were reviewed and approved by the Institutional Animal Care and Use Committee at the North China University of Science and Technology (Tangshan, China). The animals received food and water according to guidelines set by the National Institutes of Health (Bethesda, MD, USA) and were housed in an air-conditioned room with a 12-h light/dark cycle at constant temperature $\left(21^{\circ} \mathrm{C}\right)$ and $55 \%$ humidity for at least 1 week prior to the experiments.

The rats were divided into four groups according to the randomized block design, namely, a control group, model group, solvent control group and OA group, with 24 rats in each group. With the exception of the rats in the control group, the rats were induced by intratracheal instillation of $\mathrm{SiO}_{2}$ $(250 \mathrm{mg} / \mathrm{kg})$. The rats in the OA group were intragastrically administered with $\mathrm{OA}(60 \mathrm{mg} / \mathrm{kg})$ daily from the second day following $\mathrm{SiO}_{2}$ administration. The rats in the solvent control group were gavaged daily with $0.6 \%$ sodium carboxymethyl cellulose $(10 \mathrm{ml} / \mathrm{kg})$ solution, whereas the rats in the control group were gavaged with physiological saline in the same conditions for 56 consecutive days. The rats ( $n=6 /$ group) were sacrificed on days 7, 14, 28 and 56. Blood samples were collected into heparinized tubes via the abdominal aorta at the four distinct time points. The blood samples were immediately centrifuged at 3,000 $\mathrm{x} g$ for $10 \mathrm{~min}$ at room temperature, and the serum was frozen at $-80^{\circ} \mathrm{C}$ for subsequent analyses. The lung tissues were immediately perfused with physiological saline solution to removed blood cells. Then right lower lobe tissues were removed and stored at $-80^{\circ} \mathrm{C}$ for western blot analysis, and the right upper lobes were used for the determination of hydroxyproline. The left lung tissues were fixed in paraformaldehyde for the assessment of morphological changes using hematoxylin and eosin (HE) staining and Masson's staining with immunohistochemical analysis.

Analysis of the oxidation/antioxidant system. The oxidation/antioxidant system includes MDA, SOD and GSH peroxidase (GHS-Px). The MDA content and activities of SOD/GSH-Px in serum samples were assayed using MDA and SOD/GSH-Px test kits according to the manufacturer's protocol (Nanjing Jiancheng Bioengineering Institute). MDA, the end product of lipid peroxidation of cells, condenses with thiobarbituric acid to form a product with a maximum absorption at $532 \mathrm{~nm}$, therefore, the MDA content in the samples was determined by comparing the optical density (O.D.) of the samples to the standard substance. SOD and GSH-Px, two important antioxidant enzymes, can scavenge free radicals and inhibit lipid peroxidation. The activities of SOD and GSH-Px activity are expressed in units, with 1 unit defined as $50 \%$ inhibition of nitrite formation. SOD can inhibit the hydroxylamines of superoxide radical anions oxidized to form nitrite, and appear violet with a color reagent; the change in absorbance was recorded at $550 \mathrm{~nm}$. The activity of GSH-Px was determined using the disulfide-nitrobenzoic acid direct method and compared at the O.D. at $412 \mathrm{~nm}$ with the standard substance.

Analysis of the levels of TNF- $\alpha$ and TGF- $\beta 1$. The serum contents of TNF- $\alpha$ and TGF- $\beta 1$ were detected using ELISA, according to the manufacturer's protocol (Wuhan Boster Biological Technology, Ltd., Wuhan, China). The anti-rat TNF- $\alpha$ (cat. no. EK0526) or TGF- $\beta 1$ (cat. no. EK0514) specific antibody was coated on the ELISA plates. The standards and samples were pipetted into the wells and the TNF- $\alpha / \mathrm{TGF}-\beta 1$ present in the sample bound to the wells by the immobilized antibody. The liquid was removed from the wells and biotinylated anti-rat TNF- $\alpha$ or TGF- $\beta 1$ antibody (1:100 dilution, at $37^{\circ} \mathrm{C}$ for $60 \mathrm{~min}$ ) was added. Following washing away unbound biotinylated antibody, HRP-conjugated streptavidin was pipetted into the wells for $30 \mathrm{~min}$ at $37^{\circ} \mathrm{C}$. The wells were again washed, and TMB color liquid was added to the wells for $20 \mathrm{~min}$ at $37^{\circ} \mathrm{C}$, with color developing in proportion to the level of TNF- $\alpha$ (TGF- $\beta 1$ ) bound. The stop solution alters the color from blue to yellow, and the intensity of the color was measured at $450 \mathrm{~nm}$. The placental TNF- $\alpha / \mathrm{TGF}-\beta 1$ levels were calculated as $\mathrm{pg} / \mathrm{ml}$ using the standard curve.

Determination of collagen content. The content of collagen in the right upper lobe lung tissue was determined using a 
hydroxyproline assay according to the manufacturer's protocol (Nianjing Jiancheng Bioengineering Institute.). Briefly, the lung tissue samples $(80 \mathrm{mg}$ ) were hydrolyzed in boiling water for $5 \mathrm{~h}$. Following addition of the immobilized reagents, the mixture was placed in $60^{\circ} \mathrm{C}$ water for $15 \mathrm{~min}$ and centrifuged at $3,000 \times \mathrm{g}$ for $15 \mathrm{~min}$ at room temperature after cooled down. The hydroxyproline content of the supernatant was quantified by spectrophotometry at $550 \mathrm{~nm}$. The data are expressed as collagen $(\mu \mathrm{g}) /$ wet weight $(\mathrm{mg})$.

Immunohistochemical analysis of AKT1-phospho S473, $N F-\kappa B$, and collagen types I and II. The immunohistochemistry used Streptavidin-peroxidase Histostain TM-Plus kits (cat. no. SP-9000; OriGene Technologies, Inc., Beijing, China). The important stages were as follows: Paraffin-embedded sections $(5 \mu \mathrm{m})$ were deparaffinized, rehydrated and underwent removal of endogenous peroxidase with $3 \% \mathrm{H}_{2} \mathrm{O}_{2}$. Following incubation with goat serum working solution, the working solution was discarded and the tissue sections were incubated with primary antibodies against AKT1-phospho S473 (1:200; Abcam), NF-кB (1:200; Santa Cruz Biotechnology, Inc.), and collagen type I (cat. no. BA0325) and III (cat. no. BA0326; 1:200; Wuhan Boster Biological Technology, Ltd.) overnight at $4^{\circ} \mathrm{C}$, followed by the biotinylated secondary antibody at $37^{\circ} \mathrm{C}$ for $15 \mathrm{~min}$ and streptavidin-peroxidase at $37^{\circ} \mathrm{C}$ for $15 \mathrm{~min}$. Immunoreactivity was visualized with DAB (Fuzhou Maixin Biotech. Co., Ltd., Fuzhou, China). Brown color staining was considered a positive result. Sections were counterstained with hematoxylin and images were captured from six separate randomly selected fields using Olympus FV1000 (magnification x400; Olympus Inc., Center Valley, PA, USA). Quantitative analysis was performed in a blinded-manner using an automatic image analysis system at Beijing University of Aeronautics and Astronautics (Beijing, China), with the average O.D. values as quantitative indicators.

Western blot analysis of AKT1 and AKT1-phospho S473. The middle lobe of the right lung $(100 \mathrm{mg})$ was lysed with RIPA lysis buffer $(1 \mathrm{ml})$, and then centrifuged at $10,000 \times \mathrm{g}$ for $15 \mathrm{~min}$ at $4^{\circ} \mathrm{C}$. The supernatant was collected and protein content was determined using a protein assay kit (Beyotime Institute of Biotechnology, Tianjin, China). The proteins (10 $\mu \mathrm{g})$ were separated by $10 \%$ SDS-PAGE under a constant voltage of $120 \mathrm{~V}$ for $2 \mathrm{~h}$, and then transferred onto a polyvinylidene fluoride membrane at a constant electric current of $250 \mathrm{~mA}$ for $30 \mathrm{~min}$ (Bio-Rad Laboratories, Inc., Hercules, CA, USA). Following blocking with 5\% non-fat dry milk, the membranes were incubated at $4^{\circ} \mathrm{C}$ overnight with primary antibodies at the following dilution ratios: GAPDH antibody (cat. no. sc-25778, 1:2,000; Santa Cruz Biotechnology, Inc.); anti-AKT1-phospho S473 antibody (1:5,000; Abcam); anti-AKT1 antibody (1:5,000; Abcam). The membranes were then washed three times with PBST and incubated with HRP-conjugated anti-rabbit IgG antibody (cat. no. 074-1506, 1:5,000; KPL, Inc., Gaithersburg, $\mathrm{MD}, \mathrm{USA}$ ) for $2 \mathrm{~h}$ at room temperature, followed by washing with PBST. The proteins were visualized using chemiluminescence (ECL; Beyotime Institute of Biotechnology).

Statistical analysis. All data are expressed as the mean \pm standard deviation. SPSS 17.0 software (SPSS, Inc.,
Table I. Silicotic nodule grades in the lungs of rats in each group $(n=6)$.

\begin{tabular}{lcccc}
\hline & \multicolumn{4}{c}{ Nodule grade following instillation } \\
\cline { 2 - 5 } Group & 7 days & 14 days & 28 days & 56 days \\
\hline Control & 0 & 0 & 0 & 0 \\
Silicosis & 0 & I & I+-II+ & II+-III \\
Solvent & 0 & I & I+-II+ & II+-III \\
OA & 0 & 0-I & I+ & I+
\end{tabular}

OA, oleanolic acid.

Chicago, IL, USA) was used to perform statistical analyses. Multiple group comparisons were performed using one-way analysis of variance followed by pair-wise comparison with the Student's $t$-test. $\mathrm{P}<0.05$ was considered to indicate a statistically significant difference.

\section{Results}

Effect of $O A$ on histopathologic changes in the lungs. Pathological changes in the lung tissues of the rats were observed by light microscopy with $\mathrm{HE}$ and Masson's staining. As shown in Fig. 1Aa-d, the lungs of the rats in the control group, which received physiological saline, had a thin alveolar septum without significant inflammation and no obvious abnormalities shown by the HE stain. However, in the model group and solvent control group, at day 14 post-instillation, there was marked infiltration of inflammatory cells and alveolar septum thickening in the lungs, with occasional small numbers of cellular nodules (stage I; and Table I). At 28 days, primarily cellular nodules (stage I+) and fibrotic cellular nodules (stage II+) were observed. At 56 days, fibrous nodules were integrated with each other, there were more fibrotic cellular nodules (stages II+ and III). By contrast, OA treatment significantly reduced inflammatory cell infiltration and alveolar septum thickening at 14 days; the size and number of cellular nodules (stage I+) were decreased at 28 and 56 days. Masson's staining (Fig. 1Ba-c) showed: blue collagen fibers, red muscle fibers, cytoplasm and red blood cells, and brown nuclei. In the control group, there was a small quantity of blue collagen fiber around the bronchial and alveolar septum area during the investigation. In the model group and solvent control group, diffuse collagen fibers were increased and arranged irregularly in the nodules at 28 days; increased collagen deposition was present, which was arranged in concentric circles, at 56 days; lung fibrosis was aggravated. OA treatment significantly reduced collagen fibers, in small sections or small bundles. These results indicated that the silicosis model was successfully constructed and that OA exerted a significant protective effect.

Effect of OA on oxidative stress in the lungs. As shown in Table II, compared with the control group, the content of MDA in sera of the model group and solvent control group increased, peaking at 14 days, followed by a marginal decrease, although significant differences were found in the statistical analysis $(\mathrm{P}<0.05)$. However, OA treatment significantly decreased the 


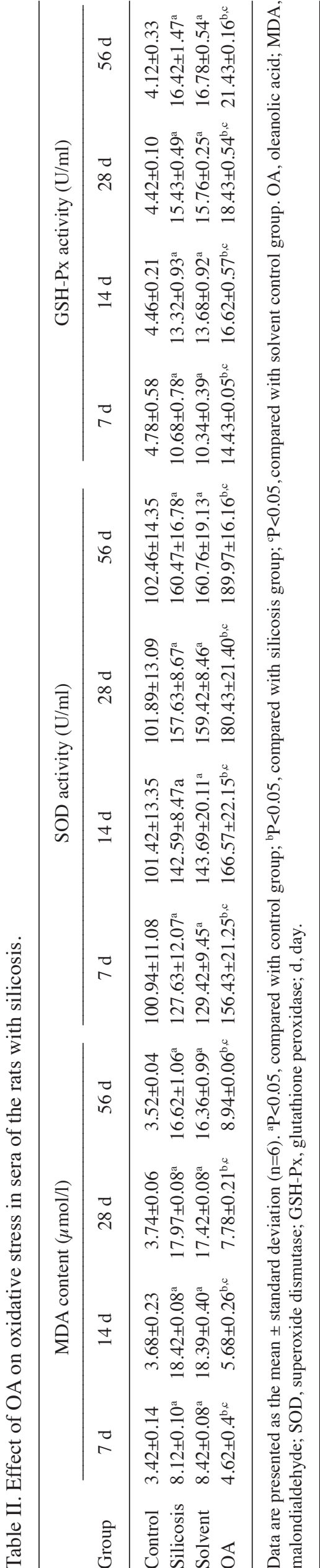
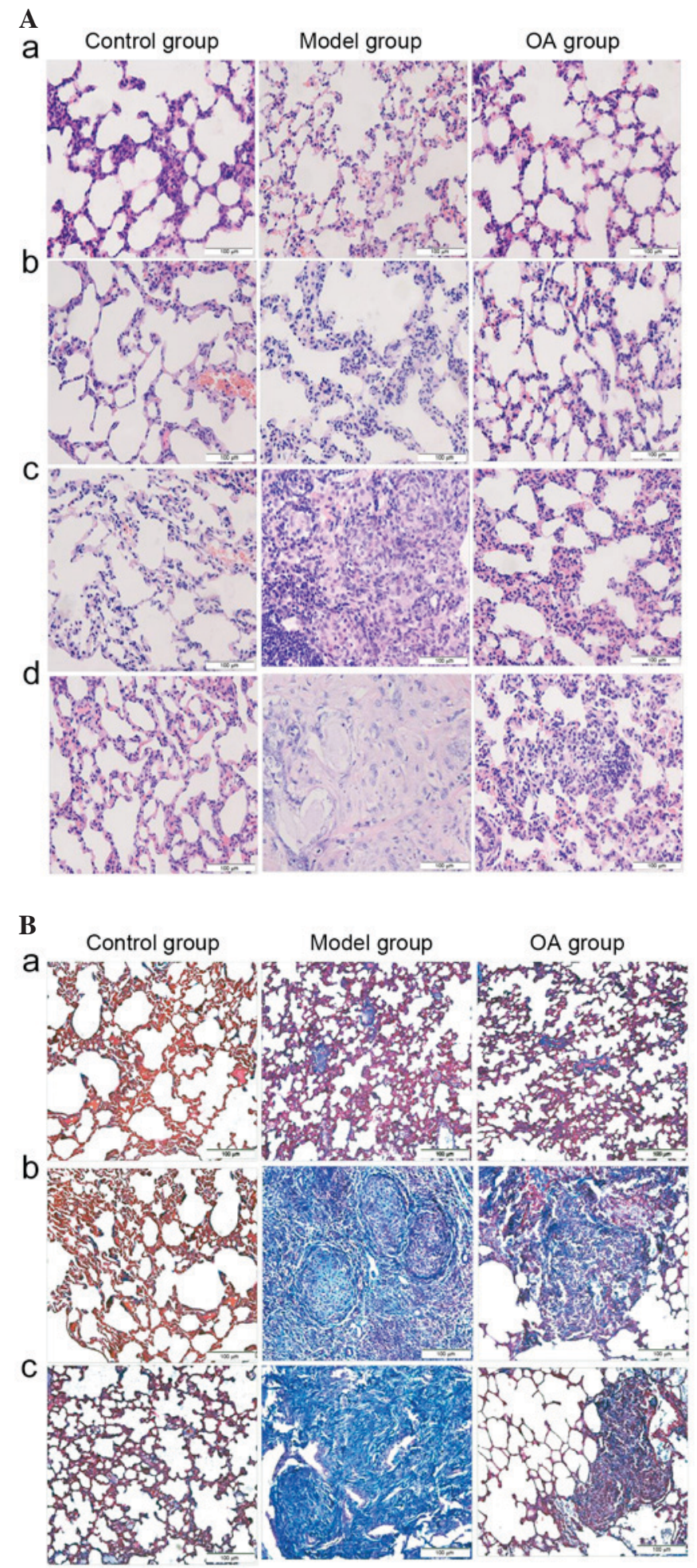

Figure 1. Morphological observation of lung tissue in each group. (A) Morphological observation of lung tissue with hematoxylin and eosin stain (magnification x 100) on (a) day 7; (b) day 14; (c) day 28; (d) day 56. (B) The morphological observations of lung tissue using Masson's stain (magnification, x100) on (a) day 14; (b) day 28; (c) day 56.

content of MDA, compared with the content in the model group and solvent control groups at corresponding time points $(\mathrm{P}<0.05)$.

The activities of SOD/GSH-Px increased in the sera from the model group and solvent control group, compared with the control group at corresponding time points $(\mathrm{P}<0.05)$, 

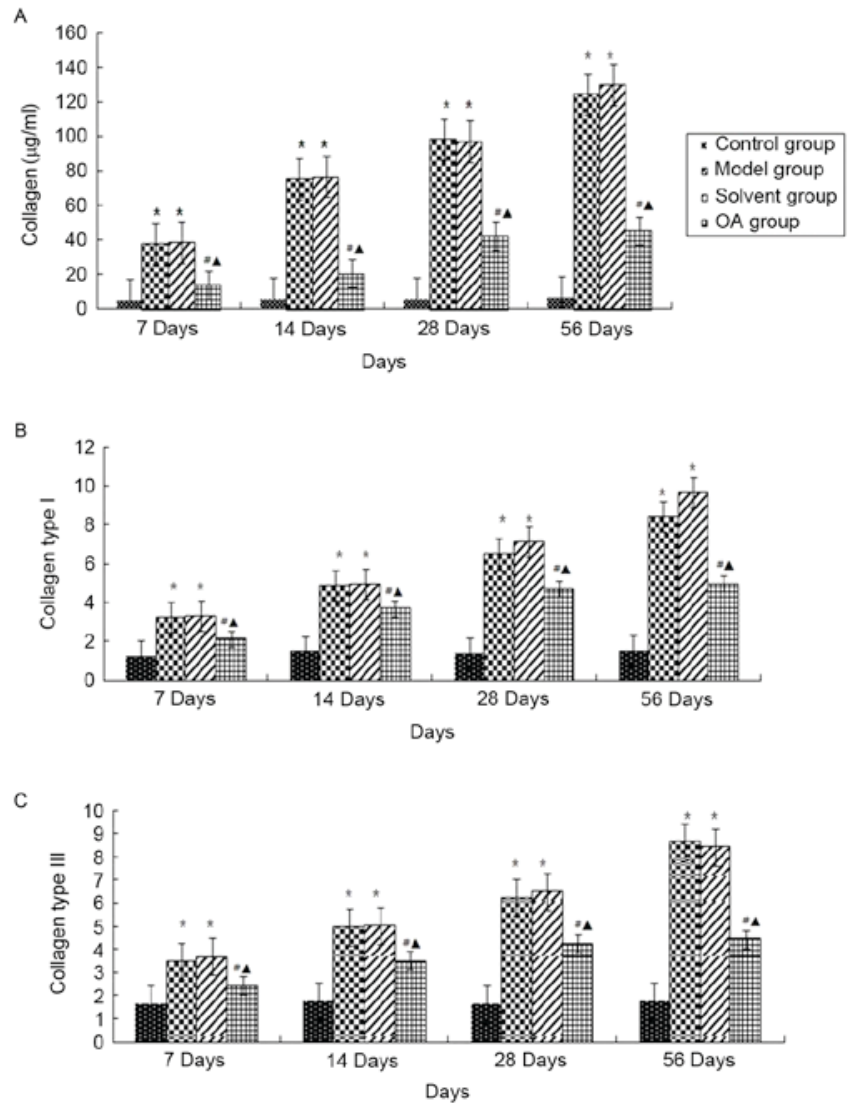

Figure 2. Effect of OA on changes in collagen in the lung. (A) Collagen; (B) collagen type I; (C) collagen type III. * $\mathrm{P}<0.05$, compared with control group; ${ }^{\text {}} \mathrm{P}<0.05$, compared with silicosis (model) group; ${ }^{\boldsymbol{\Delta}} \mathrm{P}<0.05$, compared with solvent control group. OA, oleanolic acid.

however, the activities of SOD/GSH-Px increased with time in the sera from the model group and solvent control group. Compared with the model group and solvent control group, the activities of SOD/GSH-Px were significantly increased in the OA group at the corresponding time points $(\mathrm{P}<0.05)$.

Effect of OA on collagen changes in the lungs. Hydroxyproline content is an important indicator of total collagen in lung fibrosis, which are predominantly composed of collagen types I and III. In the present study, we detected the content of hydroxyproline using a hydroxyproline kit, and the expression levels of collagen types I and III using immunohistochemistry (Fig. 2A-C). No significant differences in the hydroxyproline content or levels of collagen types I and III were found in the control group during the investigation. However, the total collagen/hydroxyproline content and the expression levels of collagen I and III in the model group and solvent control group were significantly higher, compared with those in the control group at corresponding time points $(\mathrm{P}<0.05)$. As expected, OA treatment significantly reduced these levels, compared with those in the model group and solvent control group $(\mathrm{P}<0.05)$, but remained higher, compared with those in the control group $(\mathrm{P}<0.05)$.

Effect of $O A$ on cytokines in the lungs. TNF- $\alpha$ and TGF- $\beta 1$ are important cytokines and are involved in inflammation and pulmonary fibrosis. As shown in Fig. 3A and B, no differences in the serum contents of TNF- $\alpha$ and TGF- $\beta 1$ were found in rats of the control group at the four time points $(\mathrm{P}>0.05)$. The serum contents of TNF- $\alpha$ in the model group and solvent control group were significantly increased, peaking at day 14 day post-instillation, with a subsequent marginal decrease, but with statistically significant differences at each time point, compared with those in the control group $(\mathrm{P}<0.05)$, whereas TGF- $\beta 1$ increased following instillation $(\mathrm{P}<0.05)$. No significant differences between the model group and solvent control group were found at different time points. OA treatment had inhibitory effects on the contents of TNF- $\alpha$ and TGF- $\beta 1$, compared with the model group and solvent group at the corresponding time points $(\mathrm{P}<0.05)$.

Effect of $O A$ on $p-A K T I / N F-\kappa B$ in the lungs. Immuno-histochemical methods were used to observe the expression of $\mathrm{p}-\mathrm{AKT} 1 / \mathrm{NF}-\kappa \mathrm{B}$ in the rat lung tissues. As shown in Fig. 4, the levels of NF- $\kappa \mathrm{B}$ p65 in the nuclear fractions were significantly increased in the model group and solvent control group, compared with that in the control group $(\mathrm{P}<0.05)$; whereas positive staining for p-AKT1 (Fig. 5) was primarily present in the nuclei of interstitial cells in the lung tissues of the model group and solvent control group, but not in the control group. OA treatment markedly reduced this positive staining $(\mathrm{P}<0.05)$. The immunohistochemical results were further confirmed using western blot analysis (Fig. 6), which showed that the model group and solvent control groups exhibited increased expression levels of ph-AKT1, compared with levels in the control group at the corresponding time points $(\mathrm{P}<0.05)$ and that $\mathrm{OA}$ treatment significantly weakened the expression of p-AKT1 in the rat lungs, compared with levels in the model group and solvent control group at the corresponding time points $(\mathrm{P}<0.05)$.

\section{Discussion}

In the present study, the effects of OA on silica-induced lung injury and fibrosis were examined for the first time, to the best of our knowledge. Firstly, successful establishment of the silicosis model in rats was confirmed by observing morphology and pathological changes in lung tissues with HE and Masson's staining. Subsequently, biochemical indicators, including changes in cytokines, collagen and the AKT/NF- $\kappa$ B pathway were measured in the pathogenesis of silicosis, and the effects of OA on these changes were determined. The resulting data suggested that OA possessed protective effects against silica-induced lung injury and fibrosis.

It is known that silicosis is a preventable occupational disease with no effective treatments available. In silica-induced inflammation and fibrosis, various mediators, including ROS, cytokines and growth factors released from activated alveolar macrophages, are key in the development and progression of the disease (13-15). ROS, which include hydroxyl radicals, superoxide anions, hydrogen peroxide and singlet oxygen, are generated not only at the particle surface, but also by phagocytic cells attempting to digest the silica particle. Particle-derived ROS can also react with cell-derived ROS and RNS, yielding novel toxic moieties, including peroxynitrite from $\mathrm{NO}$ and superoxide anions $\left(\mathrm{O}_{2} \bullet\right)(16)$. Therefore, oxidative stress is 

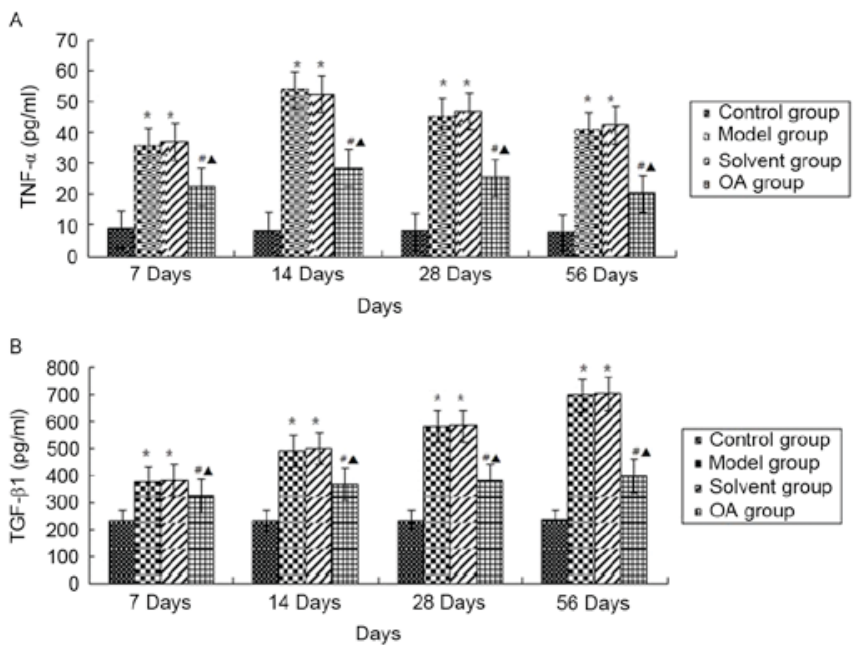

Figure 3. Effect of OA on cytokines in the lung. (A) Effect of OA on TNF- $\alpha$; (B) Effect of OA on TGF- $\beta$. " $\mathrm{P}<0.05$, compared with control group; ${ }^{\#} \mathrm{P}<0.05$, compared with silicosis (model) group; ${ }^{\mathbf{A}} \mathrm{P}<0.05$, compared with solvent control group. OA, oleanolic acid. TNF- $\alpha$, tumor necrosis factor- $\alpha$; TGF- $\beta$, transforming growth factor- $\beta$.

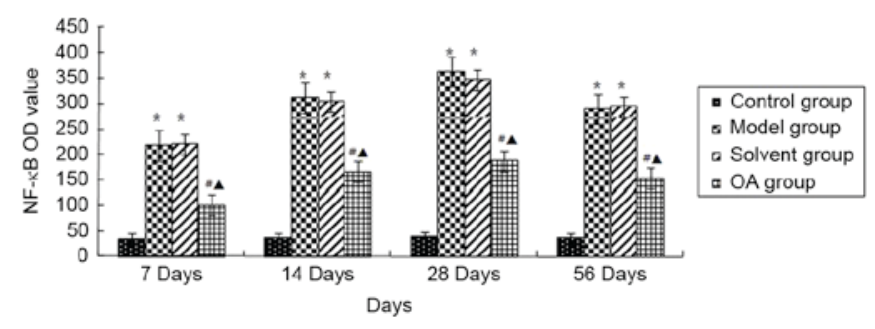

Figure 4. Effect of $\mathrm{OA}$ on $\mathrm{NF}-\kappa \mathrm{B} / \mathrm{p} 65$ in the lung. ${ }^{*} \mathrm{P}<0.05$, compared with control group; ${ }^{\#} \mathrm{P}<0.05$, compared with silicosis (model) group; ${ }^{\mathbf{\Delta}} \mathrm{P}<0.05$, compared with solvent control group. OA, oleanolic acid; NF- $\mathrm{B}$, nuclear factor- $\kappa \mathrm{B}$; OD, optical density.

caused by an imbalance between the production of ROS and the ability of the biological system to repair the resulting damage. It has been demonstrated that oxidative stress is involved in the development of silica-induced pulmonary disease in rats (8). In humans, silica exposure also activates oxidative stress in the development and progression of silicosis (17). The quantification of oxidative stress can also be assessed by the measurement of aldehydes, including MDA, the end product of lipid peroxidation of cells. The plasma levels of MDA are correlated with the severity of silicosis (18). Oxidative stress is also evidenced by increased the expression of antioxidant enzymes, including SOD and GSH-Px. SOD, a superoxide anion radical scavenger, can inhibit the peroxidation of free radicals and indirectly reflect the level of free radicals scavenged. GSH-Px can promote the removal of hydrogen peroxide $\left(\mathrm{H}_{2} \mathrm{O}_{2}\right)$ and free radicals by GSH, and is important in the integrity of cell membrane structure and function. This was supported by a study by Zhang et al (19), which found that the mean serum levels of GSH and MDA, and activity of SOD in the silicosis group were significantly higher, compared with those in control subjects without silicosis $(\mathrm{P}<0.05)$. Consistent with these results, the present study found that MDA content and the activity of SOD/GSH-Px in the sera of the model

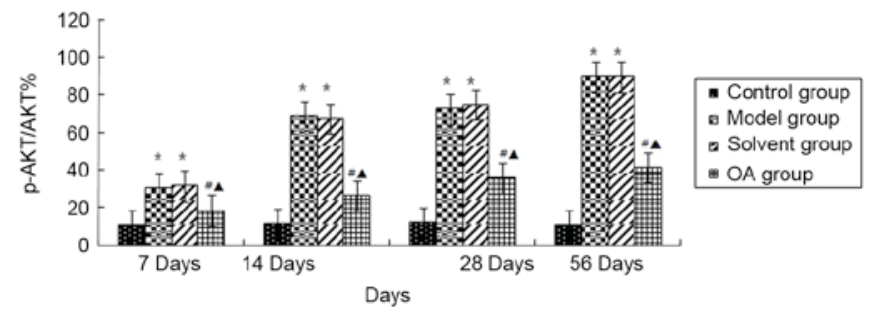

Figure 5. Effect of OA on p-AKT/AKT in the lung. ${ }^{*} \mathrm{P}<0.05$, compared with control group; ${ }^{\prime} \mathrm{P}<0.05$, compared with silicosis (model) group; ${ }^{\mathbf{\Delta}} \mathrm{P}<0.05$, compared with solvent control group. OA, oleanolic acid; $\mathrm{p}-$, phosphorylated.

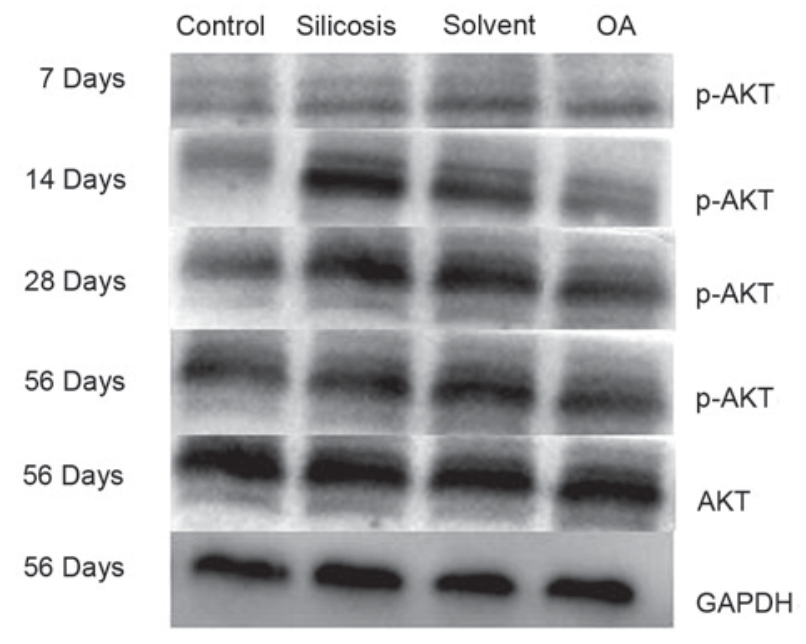

Figure 6. Effect of OA on p-AKT/AKT in the lung. Levels of p-AKT/AKT were determined using western blot analysis. OA, oleanolic acid; p-, phosphorylated.

group and solvent control group were increased, however, OA modulated these changes by downregulating MDA content and increasing the activity of SOD/GSH-Px, which suggested that OA may have an antioxidant role in the development and progression of silicosis.

Intracellular ROS has a fundamental role in the silica-induced transduction pathway leading to the production of TNF- $\alpha$. Scarfî et al found that $\mathrm{H}_{2} \mathrm{O}_{2}$ and $\mathrm{OH} \bullet$ radicals were key signals in the enhanced production of TNF- $\alpha$ in QA-stimulated RAW 264.7 murine macrophages (20). TNF- $\alpha$ is a pleiotropic cytokine, shown to be involved in inflammation and fibrosis $(21,22)$. In several studies, serum levels of TNF- $\alpha$ in silicosis groups were reported to be significantly higher, compared with those in control groups $(23,24)$. The released TNF- $\alpha$ increases the function of neutrophils and eosinophils, which generates increased superoxide and lysosomal enzyme release, and produces toxic effects to surrounding tissues to further increase the inflammatory response. TNF- $\alpha$ can also aggregation secrete abundant human TGF- $\beta 1$, promotes the proliferation of fibroblasts and secretes increased collagen (25). The results of the present study suggested that TNF- $\alpha$ was produced at an early stage of the inflammatory process in the silicosis model, peaked 14 days following instillation and remained at a higher level, compared with levels in the control group, which was consistent with the literature. Of note, OA treatment inhibited the levels of TNF- $\alpha$, compared with those 
in the model group and solvent group at the corresponding time points.

TGF- $\beta 1$, a multifunctional cytokine, regulates the proliferation and differentiation of cells (26) and is known to promote the pathogenesis of lung fibrosis. The mRNA and protein expression of TGF- $\beta 1$ have been shown to increase in the lungs of the silicotic animals $(27,28)$. Consistent with these results, the present study found that, 56 days post- $\mathrm{SiO}_{2}$ instillation, the pulmonary expression of TGF- $\beta 1$ increased almost 2.13 -fold, compared with that in the control group, which was markedly inhibited by chronic administration of OA. These data suggested that OA suppressed the expression of TNF- $\alpha$ and TGF- $\beta 1$, accordingly reducing the rate of the development and progression of silicosis.

As is already known, $N F-\kappa B$, a critical transcription factor in modifying the production of inflammatory cytokines, growth factors and ROS, is activated by silica in macrophages and other types of lung cells, and is important in the initiation and progression of silica-induced pulmonary fibrosis (29-32). Several studies have confirmed that the PI3K/Akt signaling pathway can be an upstream activator of the $\mathrm{NF}-\kappa \mathrm{B}$ signaling cascade (33) and adjust cells physiological function (34). AKT, a serine/threonine protein kinase, is activated via the PI3K pathway. Activated AKT can promote the transcriptional activity of $\mathrm{NF}-\kappa \mathrm{B}$ by accelerating the degradation of IKB and phosphorylating $\mathrm{NF}-\kappa \mathrm{B} / \mathrm{p} 65$ (35). OA has been reported to inhibit the activation and nuclear translocation of $\mathrm{NF}-\kappa \mathrm{B}$, resulting in suppression of the TNF- $\alpha$-induced inflammatory response (36). In the present study, the data showed that levels of p-Akt Ser473 and NF- $\kappa \mathrm{B} / \mathrm{p} 65$ were increased in the progression of silica-induced pulmonary fibrosis in rats, and that OA treatment downregulated the phosphorylation of AKT-Ser473 and decreased the level of $\mathrm{NF}-\kappa \mathrm{B} / \mathrm{p} 65$. These results indicated that $\mathrm{OA}$ inhibited silica-induced pulmonary inflammation and fibrosis in rats, possibly through regulating the $\mathrm{AKT} / \mathrm{NF}-\kappa \mathrm{B}$ pathway.

In conclusion, these results of the present study suggested that OA restored the oxidant/antioxidant balance, and decreased pulmonary cytokines and collagen through regulating the $\mathrm{AKT} / \mathrm{NF}-\kappa \mathrm{B}$ pathway in silica-induced lung injury and fibrosis. The protective effects of OA in silicosis may be relevant, not only during the first steps of the lung inflammatory response, but also subsequently. OA, a pentacyclic triterpenoid extensively found in a variety of plants and medicinal herbs, may be an effective option for treating silicosis, for which there are no other specific treatment options.

\section{Acknowledgements}

This study was supported by the Tangshan Science and Technology Bureau Foundation of China (grant no. 14130262B).

\section{References}

1. Rimal B, Greenberg AK and Rom WN: Basic pathogenetic mechanisms in silicosis: Current understanding. Curr Opin Pulm Med 11: 169-173, 2005.

2. Flynn MR and Susi P: Engineering controls for selected silica and dust exposures in the construction industry-a review. Appl Occup Environ Hyg 18: 268-277, 2003.
3. Leung CC, Yu IT and Chen W: Silicosis. Lancet 379: 2008-2018, 2012.

4. Thomas CR and Kelley TR: A Brief Review of silicosis in the United states. Environ Health Insights 4: 21-26, 2010.

5. Occupational Health Technical Service Network: Silicosis. http://www.zybw.com/zybw_list.aspx?c=31\&a=60\&t=xq\&m=502, Accessed: January 5, 2014.

6. Hamilton RF Jr, Thakur SA and Holian A: Silica binding and toxicity in alveolar macrophages. Free Radic Biol Med 44: 1246-1258, 2008.

7. Zhang L, He YL, Li QZ, Hao XH, Zhang ZF, Yuan JX, Bai YP, Jin YL, Liu N and Chen G: N-acetylcysteine alleviated silica-induced lung fibrosis in rats by down-regulation of ROS and mitochondrial apoptosis signaling. Toxicol Mech Methods 24: 212-219, 2014

8. Porter DW, Millecchia LL, Willard P, Robinson VA, Ramsey D, McLaurin J, Khan A, Brumbaugh K, Beighley CM, Teass A and Castranova V: Nitric oxide and reactive oxygen species production causes progressive damage in rats after cessation of silica inhalation. Toxicol Sci 90: 188-197, 2006.

9. Liu J: Oleanolic acid and ursolic acid: Research perspectives. J Ethnopharmacol 100: 92-94, 2005.

10. Pollier J and Goossens A: Oleanolic acid. Phytochemistry 77: 10-15, 2012.

11. Chung S, Yoon HE, Kim SJ, Kim SJ, Koh ES, Hong YA, Park CW, Chang YS and Shin SJ: Oleanolic acid attenuates renal fibrosis in mice with unilateral ureteral obstruction via facilitating nuclear translocation of Nrf2. Nutr Metab (Lond) 11: 2, 2014.

12. Kulkarni AA, Thatcher TH, Olsen KC, Maggirwar SB, Phipps RP and Sime PJ: PPAR- $\gamma$ Ligands Repress TGFb-induced myofibroblast differentiation by targeting the PI3K/Akt pathway: Implications for therapy of fibrosis. PLoS One 6: e15909, 2011.

13. Thakur SA, Beamer CA, Migliaccio CT and Holian A: Critical role of MARCO in crystalline silica-induced pulmonary inflammation. Toxicol Sci 108: 462-471, 2009.

14. Lim Y, Kim JH, Kim KA, Chang HS, Park YM, Ahn BY and Phee YG: Silica-induced apoptosis in vitro and in vivo. Toxicol Lett 108: 335-339, 1999.

15. Sun Y, Yang F, Yan J, Li Q, Wei Z, Feng H, Wang R, Zhang L and Zhang X: New anti-fibrotic mechanisms of n-acetyl-seryl-aspart yl-lysyl-proline in silicon dioxide-induced silicosis. Life Sci 87: 232-239, 2010

16. Fubini B and Hubbard A: Reactive Oxygen Species (ROS) and reactive nitrogen species (RNS) generation by silica in inflammation and fibrosis. Free Radic Biol Med 34: 1507-1516, 2003.

17. Palabiyik SS, Girgin G, Tutkun E, Yilmaz OH and Baydar T: Immunomodulation and oxidative stress in denim sandblasting workers: Changes caused by silica exposure. Arh Hig Rada Toksikol 64: 431-437, 2013.

18. Pelclová D, Fenclová Z, Syslová K, Vlčková S, Lebedová J, Pecha O, Běláček J, Navrátil T, Kuzma M and Kačer P: Oxidative stress markers in exhaled breath condensate in lung fibroses are not significantly affected by systemic diseases. Ind Health 49: 746-754, 2011.

19. Zhang JW, Lv GC, Yao JM and Hong XP: Assessment of serum antioxidant status in patients with silicosis. J Int Med Res 38 : 884-889, 2010.

20. Scarfì S, Magnone M, Ferraris C, Pozzolini M, Benvenuto F, Benatti $\mathrm{U}$ and Giovine M: Ascorbic acid pre-treated quartz stimulates TNF-alpha release in RAW264.7 murine macrophages through ROS production and membrane lipid peroxidation. Respir Res 10: 25, 2009.

21. Jiang ZY, Zou L, Shi SS, Lu YR, Dong J, Yang CH, Lu YC and Dai GK: Effects of curcumin on TNF-alpha and TGF-betal in serum and lung tissue of $\mathrm{SiO} 2$-induced fibrosis in mice. Xi Bao Yu Fen Zi Mian Yi Xue Za Zhi 25: 399-401, 2009 (In Chinese).

22. Li Z, Xue J, Yan S, Chen P and Chen L: Association between tumor necrosis factor- $\alpha$ 308G/A gene polymorphism and silicosis susceptibility: A meta-analysis. PLoS One 8: e76614, 2013.

23. Miao RM, Zhang XT, Yan YL, He EQ, Guo P, Zhang YY, Zhao DK, Yang ZG, Chen J, Yao MY, et al: Change of serum TGF-beta1 and TNF-alpha in silicosis patients. Zhonghua Lao Dong Wei Sheng Zhi Ye Bing Za Zhi 29: 606-607, 2011 (In Chinese).

24. Slavov E, Miteva L, Prakova G, Gidikova P and Stanilova S: Correlation between TNF-alpha and IL-12p40-containing cytokines in silicosis. Toxicol Ind Health 26: 479-486, 2010. 
25. Ortiz LA, Lasky J, Gozal E, Ruiz V, Lungarella G, Cavarra E, Brody AR, Friedman M, Pardo A and Selman M: Tumor necrosis factor receptor deficiency alters matrix metalloproteinase 13/tissue inhibitor of metalloproteinase 1 expression in murine silicosis. Am J Respir Crit Care Med 163: 244-252, 2001.

26. Xu H, Yang F, Sun Y, Yuan Y, Cheng H, Wei Z, Li S, Cheng T, Brann D and Wang R: A New Antifibrotic Target of Ac-SDKP: Inhibition of Myofibroblast Differentiation in Rat Lung with Silicosis. PLoS One 7: e40301, 2012.

27. Fan LH, Liu TF, Guo M, Liu ML, Wang ZP and Si SJ: Effect of schisandrin $\mathrm{B}$ on lung mRNA expression of transforming growth factor-betal signal transduction molecule in rat lungs exposed to silica. Zhonghua Lao Dong Wei Sheng Zhi Ye Bing Za Zhi 29: 255-259, 2011 (In Chinese).

28. Maron-Gutierrez T, Castiglione RC, Xisto DG, Oliveira MG, Cruz FF, Peçanha R, Carreira-Junior H, Ornellas DS, Moraes MO, Takiya CM, et al: Bone marrow-derived mononuclear cell therapy attenuates silica-induced lung fibrosis. Eur Respir J 37: 1217-1225, 2011.

29. Porter DW, Ye J, Ma J, Barger M, Robinson VA, Ramsey D, McLaurin J, Khan A, Landsittel D, Teass A and Castranova V: Time course of pulmonary response of rats to inhalation of crystalline silica: NF-kappa B activation, inflammation, cytokine production, and damage. Inhal Toxicol 14: 349-367, 2002.

30. Di Giuseppe M, Gambelli F, Hoyle GW, Lungarella G, Studer SM Richards T, Yousem S, McCurry K, Dauber J, Kaminski N, et al: Systemic Inhibition of NF-kappaB activation protects from silicosis. PLoS One 4: e5689, 2009.
31. Chen F and Shi X: NF-kappaB, a pivotal transcription factor in silica-induced diseases. Mol Cell Biochem 234-235: 169-176, 2002.

32. Hubbard AK, Timblin CR, Shukla A, Rincón M and Mossman BT: Activation of NF-kappaB-dependent gene expression by silica in lungs of luciferase reporter mice. Am J Physiol Lung Cell Mol Physiol 282: L968-L975, 2002.

33. Venkatesan B, Valente AJ, Prabhu SD, Shanmugam P, Delafontaine $\mathrm{P}$ and Chandrasekar B: EMMPRIN activates multiple transcription factors in cardiomyocytes, and induces interleukin-18 expression via Rac1-dependent PI3K/Akt/IKK/NF-kappaB andMKK7/JNK/AP-1 signaling. J Mol Cell Cardiol 49: 655-663, 2010.

34. Jing Y, Liu LZ, Jiang Y, Zhu Y, Guo NL, Barnett J, Rojanasakul Y, Agani $\mathrm{F}$ and Jiang BH: Cadmium Increases HIF-1 and VEGF Expression through ROS, ERK, and AKT signaling pathways and induces malignant transformation of human bronchial epithelial cells. Toxicol Sci 125: 10-19, 2012.

35. Yasuda T: Activation of Akt leading to NF- $\mathrm{NB}$ up-regulation in chondrocytes stimulated with fibronectin fragment. Biomed Res 32: 209-215, 2011.

36. Takada K, Nakane T, Masuda K and Ishii H: Ursolic acid and oleanolic acid, members of pentacyclic triterpenoid acids, suppress TNF- $\alpha$-induced E-selectin expression by cultured umbilical vein endothelial cells. Phytomedicine 17: 1114-1119, 2010. 